\title{
Effectiveness of sepsis bundle application in cirrhotic patients with septic shock: a single-center experience
}

\author{
Laura Rinaldi ${ }^{a}$, Elena Ferrari ${ }^{a}$, Marco Marietta ${ }^{b}$, Lara Donno ${ }^{a}$, Davide Trevisan ${ }^{a}$, \\ Mauro Codeluppi ${ }^{c}$, Stefano Busani ${ }^{a}$, Massimo Girardis ${ }^{a, *}$
}

\author{
${ }^{\mathrm{a}}$ Department of Anaesthesiology and Intensive Care, University of Modena and Reggio Emilia and \\ University Hospital of Modena, L.go del Pozzo, Modena, 41100, Italy \\ ${ }^{\mathrm{b}}$ Department of Haematology, University Hospital of Modena, L.go del Pozzo, Modena, 41100, Italy \\ ${ }^{\mathrm{c}}$ Department of Infectious Diseases, University Hospital of Modena, L.go del Pozzo, Modena, 41100, Italy
}

\section{Keywords: \\ Cirrhosis; \\ Intensive care; \\ Sepsis bundles; \\ Septic shock}

\begin{abstract}
Purpose: To evaluate the effect of adherence to evidence-based guidelines of the Surviving Sepsis Campaign (SSC) on the outcome of cirrhotic patients with septic shock admitted to the intensive care unit. Methods: This prospective observational cohort study included 38 patients with documented liver cirrhosis and septic shock admitted to a multidisciplinary intensive care unit at a University Hospital from January 2005 to June 2009. In each patient, the compliance to 4 resuscitation (ie, 6-hour bundle) and to 3 management (i.e. 24-hour bundle) interventions recommended by the SSC guidelines and the 30-day mortality were measured.

Results: The 6-hour, 24-hour, and all bundles were completed in $50 \%, 52 \%$, and $39 \%$ of the patients, respectively. The characteristics at admission and the 30-day mortality of patients with allbundle compliance $(\mathrm{n}=15$; mortality $86.6 \%)$ were similar to those of patients without bundle compliance $(n=23$; mortality $78.2 \%)$, except for central venous $\mathrm{O}_{2}$ saturation. Unadjusted and adjusted regression analysis showed that none of the single sepsis interventions and bundles were independently associated with 30-day mortality.

Conclusions: In our observational study, the adherence to the interventions recommended by the SSC evidence-based guidelines did not provide an improvement in the survival rate of cirrhotic patients with septic shock.
\end{abstract}

(C) 2013 Elsevier Inc. All rights reserved.

Abbreviations: SSC, Surviving Sepsis Campaign; ICU, intensive care unit; $\mathrm{PaO}_{2} / \mathrm{FiO}_{2}$, arterial O2 partial pressure to inspired O2 fraction ratio; MAP, mean arterial pressure; ALI, acute lung injury; ARDS, Adult respiratory distress syndrome; $\mathrm{ScvO}_{2}$, central venous oxygen saturation; SAPS, Simplified Acute Physiology Score; SOFA, simplified organ failure assessment; MELD, Model End-stage Liver Disease score.

* Corresponding author. Cattedra di Anestesia e Rianimazione, Azienda Ospedaliera Universitaria di Modena, L.go del Pozzo, 71, 41100 Modena, Italy. Tel.: +39059 4224896; fax: +390594224899.

E-mail address: girardis.massimo@unimo.it (M. Girardis). 


\section{Introduction}

Liver cirrhosis is one of the most frequent chronic disease over the world. In 2002, more than 300,000 adult men in the European Union have died from liver cirrhosis complications such as hemorrhage and sepsis [1]. End-stage liver disease is a well-defined risk factor for development of infections and sepsis in cirrhotic patients is frequently associated with encephalopathy, renal failure, and gastrointestinal bleeding [2-4]. The mortality rate of cirrhotic patients with sepsis greatly exceeds that of septic patients without liver disease, approaching to $100 \%$ in patients requiring intensive care unit (ICU) admission with 3 or more organ dysfunctions $[5,6]$.

In 2004 and in 2008, the guidelines of the Surviving Sepsis Campaign (SSC) [7,8] recommended a series of evidence-based interventions (ie, sepsis bundles) whose application ought to improve the outcome of patients with severe sepsis and septic shock. Numerous recently published articles showed that the application of the SSC was effective in decreasing the hospital mortality of septic shock patients $[9,10]$, and this was the case also in our experience [11]. However, the effectiveness of these interventions has never been assessed in population with specific comorbidities that can interfere with the normal inflammatory responses to infection, as for instance, liver cirrhosis. In this study we evaluated the effects of the sepsis interventions proposed by SSC guidelines on the all-cause 30-day mortality in cirrhotic patients with septic shock admitted to the intensive care unit (ICU).

\section{Methods}

\subsection{Patients}

This prospective observational study enrolled patients with liver cirrhosis and septic shock admitted from January 2005 to June 2009 to ICU of a University Hospital with a liver transplantation program. The study was approved by the local ethical committee. Most patients $(90 \%)$ were in waiting list for liver transplantation and had histological documentation and/or a clear medical history of chronic liver failure. The patient was considered to be in septic shock if all the following conditions were satisfied: (i) mean arterial pressure was less than $60 \mathrm{mmHg}$ despite adequate fluid resuscitation, (ii) there were signs of tissue hypoperfusion (eg, oliguria, acidosis, worsening of mental status), (iii) there was evidence of systemic inflammatory response syndrome (eg, body temperature $>38^{\circ} \mathrm{C}$ or $<36^{\circ} \mathrm{C}$; tachycardia, tachypnea, leukocytosis, or leukopenia), and (iv) there was a microbiologically documented infection. Patients with shock of uncertain etiology and with do not resuscitate order or end-life decisions were not included in the study. Only the first episode of septic shock was considered for each patient.

\subsection{Data collection}

In each patient, the compliance to 4 resuscitation interventions (6-hour bundle) and 3 management interventions (24-hour bundle) was evaluated. The 6-hour bundle includes (1) blood cultures collection before antibiotic administration and (2) empiric antibiotic therapy within 3 hours from diagnosis, adequate fluid resuscitation before vasopressor administration, and central venous oxygen saturation $\left(\mathrm{ScvO}_{2}\right)$ optimization within 6 hours $\left(\mathrm{ScvO}_{2}>70 \%\right)$. The 24-hour bundles includes: (1) blood glucose median $<150 \mathrm{mg} / \mathrm{dL}$ in the first 24 hours, (2) low-dose hydrocortisone administration in association with vasopressor support, (3) plateau inspiratory pressure $<30 \mathrm{~cm} \mathrm{H}_{2} \mathrm{O}$ in patients with acute lung injury/adult respiratory distress syndrome. The term adequate fluid resuscitation indicates a central venous pressure $>6 \mathrm{~mm} \mathrm{Hg}$ $(>8 \mathrm{~mm} \mathrm{Hg}$ if mechanically ventilated) or a global end-diastolic volume by trans-pulmonary thermodilution (PiCCO system, Pulsion, Germany) $>700 \mathrm{~mL} / \mathrm{m}^{2}$. The use of recombinant human activated protein $\mathrm{C}$ was not considered because of its contraindications in patients with chronic liver disease.

Two of the authors (L.R. and L.D), not involved in the management of the patients, collected the above interventions by analysis of clinical charts, and any uncertain data were reviewed with the attending physician. The interventions were classified in a dichotomous way, that is, completed or not completed. If an intervention was not applied because it not applicable (eg, low inspiratory pressure in patient without acute lung injury/adult respiratory distress syndrome), it was defined as completed. The time zero for bundles timing was the time in which signs of septic shock were documented by clinical notes. Grade of sepsis, primary site of infection, SAPS II and SOFA scores [12,13], and 30-day mortality were also recorded for each patient. The grade of liver failure was assessed at ICU admission using the Model End-Stage Liver Disease (MELD) score [14].

\subsection{Statistical analysis}

$\chi^{2}$ Test and ANOVA single-factor analysis were used when appropriate. Kaplan-Meier curves and log-rank tests were used for the comparison of 30-day survival curves between patients with and without sepsis bundles completion. To estimate the independent effect of each single intervention and bundle on 30-day mortality, univariate unadjusted and multivariate logistic regression adjusted for age, MELD, and SOFA scores were used. Data analyses were performed by means of SPSS version 15.0 (SPSS, Chicago, IL) and $P<.05$ was considered significant.

\section{Results}

Thirty-eight patients with liver cirrhosis and septic shock were studied (Table 1). Apart from steroids administration 
Table 1 Main characteristics and laboratory data at ICU admission in all the study population and in patients with and without all-bundle completion

\begin{tabular}{|c|c|c|c|}
\hline & $\begin{array}{l}\text { All } \\
\text { patients }\end{array}$ & $\begin{array}{l}\text { Bundles } \\
\text { completed }\end{array}$ & $\begin{array}{l}\text { Bundles not } \\
\text { completed }\end{array}$ \\
\hline Patients (n) & 38 & 15 & 23 \\
\hline Age (years; mean $\pm \mathrm{SD}$ ) & $51 \pm 10$ & $50 \pm 12$ & $52 \pm 10$ \\
\hline Female $(\%)$ & 29 & 27 & 30 \\
\hline \multicolumn{4}{|l|}{ Cirrhosis etiology } \\
\hline Viral, n (\%) & $28(74)$ & $12(80)$ & $16(70)$ \\
\hline Alcoholic, n (\%) & $6(16)$ & $1(7)$ & $5(22)$ \\
\hline Other, $\mathrm{n}(\%)$ & $4(10)$ & $2(13)$ & $2(9)$ \\
\hline MELD score $($ mean $\pm \mathrm{SD})$ & $35 \pm 12$ & $39 \pm 11$ & $33 \pm 12$ \\
\hline \multicolumn{4}{|l|}{ Site of infection } \\
\hline Pneumonia, n (\%) & $25(66)$ & $11(73)$ & $14(61)$ \\
\hline $\begin{array}{l}\text { Intra-abdominal infection, } \\
\mathrm{n}(\%)\end{array}$ & $15(39)$ & $6(43)$ & 9 (39) \\
\hline Blood, n (\%) & $21(55)$ & $7(47)$ & $14(61)$ \\
\hline Urinary tract, $\mathrm{n}(\%)$ & $16(42)$ & $7(47)$ & $9(39)$ \\
\hline SAPS II (mean \pm SD) & $67 \pm 20$ & $68 \pm 16$ & $67 \pm 22$ \\
\hline SOFA $($ mean \pm SD) & $16 \pm 3$ & $17 \pm 2$ & $16 \pm 3$ \\
\hline $\begin{array}{l}\text { Prothrombin time } \\
\quad(\text { INR, mean } \pm \mathrm{SD})\end{array}$ & $3.1 \pm 1.4$ & $3.1 \pm 1.4$ & $3.1 \pm 1.4$ \\
\hline $\begin{array}{l}\text { Bilirubin }(\mathrm{mg} / \mathrm{dL} \\
\text { mean } \pm \mathrm{SD})\end{array}$ & $20 \pm 15$ & $25 \pm 14$ & $17 \pm 15$ \\
\hline $\begin{array}{l}\text { Creatinine }(\mathrm{mg} / \mathrm{dL} \text {, } \\
\text { mean } \pm \mathrm{SD})\end{array}$ & $2.9 \pm 2$ & $3.7 \pm 2.2$ & $2.5 \pm 1.8$ \\
\hline RRT (n, \%) & $9(24)$ & $4(27)$ & $5(22)$ \\
\hline $\begin{array}{l}\text { Lactate }(\mathrm{mg} / \mathrm{dL} \\
\quad \text { mean } \pm \mathrm{SD})\end{array}$ & $60 \pm 47$ & $61 \pm 35$ & $60 \pm 54$ \\
\hline $\mathrm{ScvO}_{2}(\%)$ & $78 \pm 11$ & $84 \pm 7 *$ & $76 \pm 12$ \\
\hline $\begin{array}{l}30 \text { day mortality, } \\
\mathrm{n}(\%)\end{array}$ & $\begin{array}{l}31 \\
(81.6)\end{array}$ & $13(86.6)$ & $18(78.2)$ \\
\hline $\begin{array}{l}\text { Hospital mortality, } \\
\mathrm{n}(\%)\end{array}$ & $\begin{array}{l}32 \\
(84.2)\end{array}$ & $13(86.6)$ & $19(82.6)$ \\
\hline
\end{tabular}

(58\%), all the single interventions were completed in more than $75 \%$ of the patients; the compliance to interventions slightly increased during the study period and the 6-hours and 24-hours bundles were completed in $50 \%$ and $52 \%$ of the patients, respectively (Fig. 1). The median time from shock presentation to administration of broad spectrum antibiotic was $58 \mathrm{~min}$ (interquartile range, 46-115 min ). Before shock appearance, 24 patients (63\%) were in antibiotic therapy that was modified in 20 of these patients after shock occurrence. Patients with compliance to all bundles $(39 \%)$ were quite similar to patients without compliance, except $\mathrm{ScvO}_{2}$ that was higher $(P<.05)$ in the compliance group. Indeed, in this group, MELD score, bilirubin, creatinine, and 30-day mortality were also larger than in no-compliance group, but not so significantly (Table 1). The 7 patients survived at 30 days had better severity scores and laboratory data at ICU admission than those observed in no survivors, whereas the compliance to 6hour and 24-hour bundles was similar (Tables 2 and 3). Kaplan-Maier plots indicated that the probability of remaining alive was similar in patients with and without bundles compliance (Fig. 2). The univariate unadjusted (Table 3) and the multivariate logistic regression analysis adjusted for MELD and SOFA scores showed that the compliance to the single treatment included in the bundles nor the compliance to 6-hour, 24-hour, and all bundles together were independently associated with 30-days mortality.

\section{Discussion}

This observational study indicated that the 30-day mortality of cirrhotic patients with septic shock admitted to ICU is extremely high and that the application of the interventions recommended by SSC guidelines does not seem to improve the survival rate in this population.

Due to their immunocompromised condition, cirrhotic patients have a high risk of developing infections [3,4]. Sepsis occurs in approximately $40 \%$ of cirrhotic hospitalized patients with an associated mortality rate significantly larger than in no-cirrhotic patients $[5,6,14]$. The evidence based treatments proposed in the SSC guidelines and the

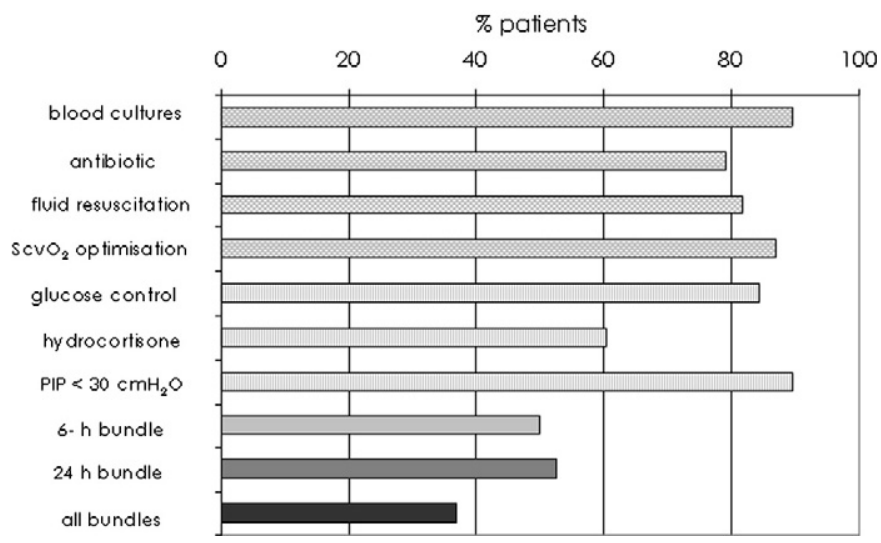

Fig. 1 Percentage of patients with compliance to each single intervention and to 6 hours, 24 hours, and all the bundles. For abbreviations and details, see text. 
Table 2 Main characteristics and laboratory data at ICU admission in survivors and no survivors at 30 days

\begin{tabular}{lll}
\hline & Survivors & No survivors \\
\hline Patients (n) & 7 & 31 \\
Age (years; mean \pm SD) & $53 \pm 13$ & $51 \pm 10$ \\
Female (\%) & 29 & 29 \\
Cirrhosis etiology & & \\
Viral, n (\%) & $4(57)$ & $21(68)$ \\
Alcoholic, n (\%) & $1(14)$ & $5(16)$ \\
Other, n (\%) & $2(28)$ & $5(16)$ \\
MELD score (mean \pm SD) & $22 \pm 9$ & $38 \pm 10 *$ \\
Site of infection & & \\
Pneumonia, n (\%) & $5(71)$ & $20(64)$ \\
Intra-abdominal infection, n (\%) & $2(28)$ & $13(42)$ \\
Blood (n, \%) & $4(57)$ & $17(55)$ \\
Urinary tract (n, \%) & $2(28)$ & $14(45)$ \\
SAPS II (mean \pm SD) & $47 \pm 13$ & $71 \pm 19 *$ \\
SOFA (mean \pm SD) & $12 \pm 4$ & $16 \pm 3 *$ \\
Prothrombin Time (INR, mean \pm SD) & $1,8 \pm 0,4$ & $3,5 \pm 1,3 *$ \\
Bilirubin (mg/dL, mean \pm SD) & $14 \pm 16$ & $21 \pm 14$ \\
Creatinine (mg/dL, mean \pm SD) & $1,0 \pm 0,42$ & $3,5 \pm 2,0 *$ \\
RRT (n, \%) & $0(0)$ & $9(29)$ \\
Lactate (mg/dL, mean \pm SD) & $19 \pm 12$ & $72 \pm 47 *$ \\
ScvO $(\%)$ & $82 \pm 7$ & $77 \pm 12$ \\
\hline
\end{tabular}

RRT, renal replacement therapy before ICU admission. $* P<.05$ compared to survivors.

management strategy based on bundles of care were effective in improving the survival rate of patients with severe sepsis [7-11]. Indeed, the effects of these treatments in patients with end-stage liver disease have never been assessed, except for low dose steroids. Fernadez et al [15] demonstrated that adrenal insufficiency is common in cirrhotic patients with severe sepsis and that the administration of hydrocortisone was associated with high frequency of shock resolution and high survival rate. In our study hydrocortisone was administered in around $60 \%$ of the patients but its use did not modify the survival rate. This discrepancy can be justified, at least in part, by differences in the studied populations: the MELD score of our patients calculated on ICU admission ( $36 \pm 12)$ exceeds the values observed by Fernandez et al (ranging between 13 and 28) [15]. Moreover, a recent randomized control trial indicated that in patients with cirrhosis and septic shock the hydrocortisone therapy was associated with hemodynamic improvement but did not reduce mortality and was associated with an increase in shock relapse and gastrointestinal bleeding [16].

In our patients, the 30-day mortality rate remained extremely high and did not depend on application of sepsis bundles. These negative results could be explained, in our opinion, by the following reasons. First, we hypothesize, in accordance with others [17], that some of the resuscitation and management interventions proposed by the SSC guidelines could be inadequate in cirrhotic patients. For instance, it is well known that the central venous $\mathrm{SO} 2$ in patients with end-stage liver disease is higher than normal because of the vasodilated hyperdynamic state. Therefore, the $\mathrm{ScvO}_{2}$ cut off value of $70 \%$ proposed by SSC guidelines could be inappropriate in this context. Likewise, the use of central venous pressure (CVP) value alone as target for fluid resuscitation could be misleading. In fact, the typical hypoalbuminemic and vasodilated state of cirrhotic patients can require a very large fluid infusion before achieving the suggested CVP values with an increased risk of interstitial and pulmonary edema. Moreover, a rapid increase of CVP values in cirrhotic patients can cause an additional risk in relation to the increase of portal pressure with bleeding complications and large ascites formation [17]. For the above reasons, in our protocols for the management of cirrhotic patients with sepsis, we decided to lower the CVP threshold before vasoactive amine administration to $6 \mathrm{mmHg}$ with respect to $8 \mathrm{mmHg}$ proposed by the SSC guidelines $[7,8]$.

Second, the identification of severe sepsis in a cirrhotic patient may be very challenging as many of the clinical signs

Table 3 Odds ratio obtained by unadjusted univariate logistic regression for 30-day mortality for each treatment completed in the 6- and 24-hour bundles

\begin{tabular}{|c|c|c|c|c|}
\hline & $\begin{array}{l}\text { Survivors }(\mathrm{n}=7) \text {, } \\
\text { completed, } \mathrm{n}(\%)\end{array}$ & $\begin{array}{l}\text { No survivors }(\mathrm{n}=31) \text {, } \\
\text { completed, } \mathrm{n}(\%)\end{array}$ & OR $(95 \% \mathrm{CI})$ & $P$ \\
\hline \multicolumn{5}{|l|}{ Single treatment } \\
\hline Blood culture & $6(86)$ & $28(90)$ & $1.11(0.98-1.25)$ & .38 \\
\hline Antibiotic $<3 \mathrm{~h}$ & $4(57)$ & $26(83)$ & $3.90(0.66-22.05)$ & .12 \\
\hline Fluid resuscitation & $5(71)$ & $28(90)$ & $3.73(0.49-18.32)$ & .18 \\
\hline ScvO2 optimization & $5(71)$ & $26(83)$ & $2.80(0.31-13.88)$ & .44 \\
\hline Glycemia control & $6(86)$ & $26(83)$ & $0.87(0.11-8.08)$ & .88 \\
\hline Steroids & $4(57)$ & $19(61)$ & $1.19(0.22-6.19)$ & .52 \\
\hline Plateau pressure control & $6(86)$ & $27(87)$ & $1.56(0.13-11.60)$ & .72 \\
\hline \multicolumn{5}{|l|}{ Bundles } \\
\hline $6 \mathrm{~h}$ & $2(28)$ & $18(51)$ & $8.31(0.89-27.50)$ & .07 \\
\hline $24 \mathrm{~h}$ & $3(42)$ & $17(54)$ & $1.62(0.31-8.48)$ & .57 \\
\hline All bundles & $2(28)$ & $13(42)$ & $4.33(0.46-20.46)$ & .17 \\
\hline
\end{tabular}



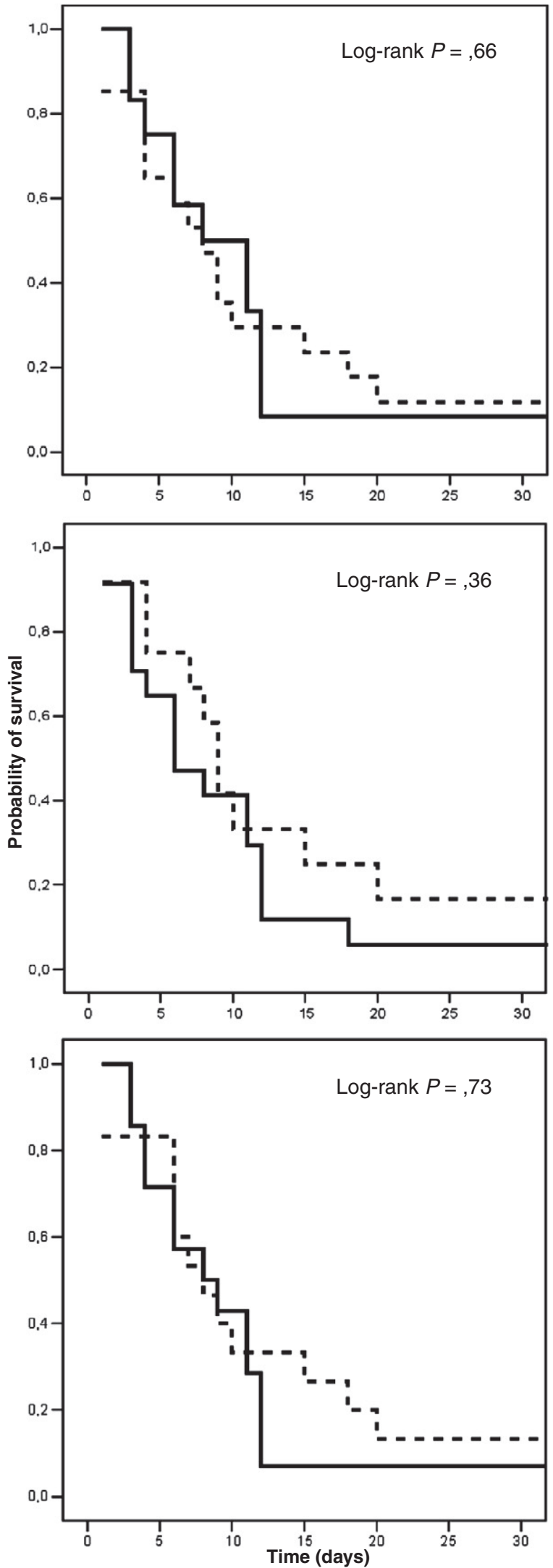

Fig. 2 Probability of survival in patients with (solid line) and without (dotted line) compliance to all interventions (upper panel), 6-hour resuscitative bundle (middle panel), and 24-hour management bundle (lower panel). of Systemic Inflammatory Response Syndrome and organ dysfunction can be present also in a cirrhotic patients without infection. For instance, hepatopulmonary and hepatorenal syndromes often complicate the clinical course of cirrhotic patients, leading to the development of severe respiratory and renal dysfunctions [17]. To avoid possibility of uncertain diagnosis, we included only cirrhotic patients with shock who had a microbiologically documented infection. The time of shock presentation was considered the time zero for evaluating the application of sepsis bundles. This could have caused a time delay between the application of the recommended sepsis interventions and the onset of severe sepsis. Therefore, it remains uncertain if an earlier application (ie, before shock) of sepsis bundles would be beneficial in these patients.

At ICU admission, the extent of organ dysfunctions in patients who survived were less severe than in dead patients: plasmatic creatinine was within normal range while lactate blood concentration and prothrombin time were only slightly altered (Table 2). The high discriminative power of renal function and SOFA score as independent variables in predicting hospital mortality of critically ill cirrhotic patients has been recently demonstrated [5]; as well, the strong correlation between lactate blood concentration, organ dysfunction and mortality in septic shock patients is well known. These observations support the hypothesis that a delay may have occurred in some patients before ICU admission and the initiation of specific sepsis therapies.

Lastly, the low number of patients included, the high compliance to sepsis interventions (ie, $>75 \%$ ), and the design of the study (ie, observational) led to unavoidable limitation, particularly in case of patients subgrouping and model analysis. In fact, at ICU admission, the patients with bundle compliance had clinical picture more severe (see Table 1) than patients without compliance. This difference further hinders definitive conclusions on the effectiveness of SSC guidelines in our patients.

In conclusion, the cirrhotic patient with septic shock is a challenge in the ICU because of its high clinical complexity and its high mortality rate. In our small cohort of cirrhotic patients with septic shock, the full application of interventions recommended by the SSC did not modify the 30-day mortality. We believe that the time of application and the targets of the recommended interventions as well as new specific therapeutic options (eg, artificial liver support) should be the objective of future clinical trials in patients with end-stage liver disease and septic shock.

\section{References}

[1] Bosetti C, Levi F, Lucchini F, Zatonski WA, Negri E, La Vecchia C. Worldwide mortality from cirrhosis: an update to 2002. J Hepatol 2007;46:827-39.

[2] Alberti C, Brun-Buisson C, Burchardi H, et al. Epidemiology of sepsis and infection in ICU patients from an international multicentre cohort study. Intensive Care Med 2002;28:108-21. 
[3] Wasmuth HE, Kunz D, Yagmur E, et al. Patients with acute on chronic liver failure display ‘sepsis-like' immune paralysis. J Hepatol 2005;42: 195-201.

[4] Wong F, Bernardi M, Balk R, et al. Sepsis in cirrhosis: report on the 7th meeting of the international ascites club. Gut 2005;54:718-25.

[5] Austin MJ, Shawcross DL. Outcome of patients with cirrhosis admitted to intensive care. Curr Opin Crit Care 2008;14:202-7.

[6] Jenq CC, Tsai MH, Tian YC, et al. RIFLE classification can predict short-term prognosis in critically ill cirrhotic patients. Intensive Care Med 2007;33:1921-30.

[7] Dellinger RP, Carlet JM, Masur H, et al. Surviving Sepsis Campaign: guidelines for management of severe sepsis and septic shock. Crit Care Med 2004;32:858-73.

[8] Dellinger RP, Levy MM, Carlet JM, et al. Surviving Sepsis Campaign: international guidelines for management of severe sepsis and septic shock. Crit Care Med 2008;36:296-327.

[9] Ferrer R, Artigas A, Levy MM, et al. Improvement in process of care and outcome after a multicenter severe sepsis educational program in Spain. JAMA 2008;299:2294-303.

[10] Ferrer R, Artigas A. Effectiveness of treatment for severe sepsis: data from the bundle improvement program. Minerva Anestesiol 2011;77: $360-5$.
[11] Girardis M, Rinaldi L, Donno L, et al. Effects on management and outcome of severe sepsis and septic shock patients admitted to the intensive care unit after implementation of a sepsis program: a pilot study. Crit Care 2009;13:R143.

[12] Le Gall JR, Lemeshow S, Saulnier F. A new Simplified Acute Physiology Score (SAPS II) based on a European/North American multicenter study. JAMA 1993;270:2957-63.

[13] Vincent JL, Moreno R, Takala J, et al. The SOFA (Sepsis-related Organ Failure Assessment) score to describe organ dysfunction/failure. Intensive Care Med 1996;22:707-10.

[14] Kamath PS, Wiesner RH, Malinchoc M, et al. A model to predict survival in patients with end-stage liver disease. Hepatology 2001;33: 464-70.

[15] Fernández J, Escorsell A, Zabalza $M$, et al. Adrenal insufficiency in patients with cirrhosis and septic shock: effect of treatment with hydrocortisone on survival. Hepatology 2006;44: 1288-95.

[16] Arabi YM, Aljumah A, Dabbagh O, et al. Low-dose hydrocortisone in patients with cirrhosis and septic shock: a randomized controlled trial. CMAJ 2010;182:1971-7.

[17] Canabal JM, Kramer DJ. Management of sepsis in patients with liver failure. Curr Opin Crit Care 2008;14:189-97. 